Pacific Journal of Mathematics

ON CERTAIN SEQUENCES OF LATTICE POINT

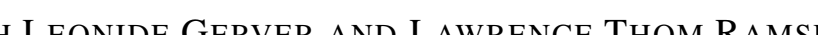




\title{
ON CERTAIN SEQUENCES OF LATTICE POINTS
}

\author{
Joseph L. Gerver and L. Thomas Ramsey
}

Let $S$ be a finite subset of $\boldsymbol{R}^{n}$. A sequence $\left\{z_{i}\right\}$ is an $S$-walk if and only if $z_{i+1}-z_{i}$ is an element of $S$ for all $i$. In an effective manner it is shown that long $S$-walks in $Z^{2}$ must have an increasing number of collinear points. In $Z^{3}$, however, an infinite $S$-walk may have a bounded number of collinear points.

1. Introduction. Let $S$ be a finite subset of $\boldsymbol{R}^{n}$.

Definition. An $S$-walk is any (finite or infinite) sequence of vectors in $\boldsymbol{R}^{n}$, say $\left\{z_{i}\right\}$, such that $z_{i+1}-z_{i} \in S$, for all $i$.

Given $S$, let $M$ be the maximum of the Euclidean norms of the vectors in $S$. In [5] the following theorem is proved (see also [3] for the case $M=\sqrt{2}$ ):

THEOREM. Let $S \subset Z^{2}$, and let $K$ be any positive integer. There exists $N=N(K, M)$ such that any $S$-walk of length at least $N$ must have $K$ collinear points.

With Theorem 1 of this paper we provide an effective bound on $N(K, M)$. With Theorem 2 we show that the situation of $S \subset Z^{3}$ is quite different, i.e., an infinite $S$-walk in $Z^{3}$ may have a bounded number of collinear points. In Theorem 3 we show that there are still some restrictions in $Z^{3}$, namely that if $S$ has only three elements, then a sufficiently long $S$-walk must have three collinear points.

\section{The Planar case.}

THeOREM 1. Let $S \subset Z^{2}$, let $K$ be any positive integer, and let $N$ be a positive integer such that

$$
\log _{2} N \geqq 2^{13} M^{4}(K-1)^{4}+\log _{2}(K-1) .
$$

Then, for every $S$-walk $\left\{z_{i}\right\}_{i=0}^{N}$, there is some line $L$, and $K$ choices for $i$, such that $z_{i} \in L$.

Proof. We suppose that the theorem is false for some $K$ and derive a contradiction. Let $Q=8 \cdot 2^{1 / 2} \cdot M(K-1)$. Let $T$ denote the set of (positive and negative) Farey fractions of order no greater than $Q$. Let $A$ be the set of all lines through the origin with 
slopes in $T$. Let $B$ be the mirror image of $A$ reflected through the line $y=x$. Enumerate the lines in the two sets $A$ and $B$ in order of increasing slope: $L_{1}, L_{2}, L_{3}, \cdots$. Let $\left\{z_{i}\right\}$ be a counterexample to the theorem for $K$. We may assume that $z_{0}$ is the origin.

Let $z_{J}$ be an arbitrary point of the counterexample sequence. There are lines in the set $A \cup B, L_{n}$ and $L_{n+1}$, such that $z_{J}$ is on or between these lines; that is, the slope of the line through the origin and $z_{J}$ is between or equal to the slopes of $L_{n}$ and $L_{n+1}$, respectively $a$ and $b$.

Dirichlet's theorem [2, page 1] gives us for $x=(a+b) / 2$, integers $p$ and $q, 0<q<Q$, such that

$$
|q x-p| \leqq Q^{-1} .
$$

We have either $p / q \geqq b \geqq a$, or $b \geqq a \geqq p / q$. Note that $a b \geqq 0$. We may therefore choose $p / q$ to be the same sign as $a$ and $b$. Let $H_{0}$ be the line through the origin with slope $p / q$ and let $U$ be the larger of the two angles between $H_{0}$ and $L_{n}$ and between $H_{0}$ and $L_{n+1}$. Clearly, since $a, b$, and $p / q$ have the same sign (viewing zero as positive and negative), the tangent of $U$ is at most $2 Q^{-1} q^{-1}$.

Enumerate the lines parallel to $H_{0}$ through points of $Z^{2}$ as $\cdots H_{-2}, H_{-1}, H_{0}, H_{1}, H_{2}, \cdots$ so that the distance from $H_{0}$ to $H_{i}$ is $|i d|$, where $d$ is the minimum distance between such translates of $H_{0}$.

We now return to $z_{J}$. Among $z_{J}, z_{J+1}, \cdots, z_{J+(2 P-1)(K-1)}$ at least one point is on some $H_{i}$ with $|i|>P-1$. Otherwise one of the $H_{i}$, with $|i| \leqq P-1$, would contain $K$ points of our $S$-walk, contrary to hypothesis. Let $z_{f}$ be on a line $H_{i}$, with $|i|>P-1$, and $J \leqq f \leqq J+(2 P-1)(K-1)$. This point $z_{f}$ is at least distance $P d$ from $H_{0}$. The component of $z_{f}$ parallel to $H_{0}$ is at most $f M$. Thus, if $V$ is the angle between $z_{f}$ and $H_{0}$, we have

$$
|\tan V| \geqq P d / f M .
$$

By taking $P$ so that $(2 P-1)(K-1) \geqq J$, we can write that

$$
\begin{aligned}
|\tan V| & \geqq P d / M[J+(2 P-1)(K-1)] \\
& \geqq P d / 2 M(2 P-1)(K-1) \\
& >d / 4 M(K-1) .
\end{aligned}
$$

We now estimate $d$. We may assume that both $L_{n}$ and $L_{n+1}$ are in $A$, since otherwise they are both in $B$ and the mirror image of the forthcoming analysis applies. With this assumption both $a$ and $b$ are in $T$. We may also assume that $p / q$ is in $T$, for if not either $p / q \geqq 1$ or $p / q \leqq-1$. In the first case $1 / 1$ will play the role of $p / q$ and in the second, $-1 / 1$. Thus 


$$
d \geqq\left(p^{2}+q^{2}\right)^{-1 / 2} \geqq\left(2^{1 / 2} q\right)^{-1} .
$$

Thus

$$
|\tan V|>\left[4 \cdot 2^{1 / 2} M q(K-1)\right]^{-1} .
$$

It is now clear that the choice of $Q$ as $8 \cdot 2^{1 / 2} M(K-1)$ gives us

$$
|\tan V|>|\tan U| \text {. }
$$

It is clear that the broken line path from $z_{J}$ to $z_{f}$ has crossed either $L_{n}$ or $L_{n+1}$. In summary, given $z_{J}$ on or between $L_{n}$ and $L_{n+1}$, there is some integer $t$ such that $0<t \leqq(2 P-1)(K-1)$ and

(i) $P$ is the first integer such that $(2 P-1)(K-1) \geqq J$ and

(ii) $z_{J+t}$ is within $M$ of either $L_{n}$ or $L_{n+1}$.

By induction we choose a subsequence $\left\{z_{t_{i}}\right\}$ of $\left\{z_{i}\right\}$ such that

(i) each $z_{t_{i}}$ is within $M$ of some line in $A \cup B$ and

(ii) $t_{i}<t_{i+1} \leqq t_{i}+(2 P-1)(K-1)$, where $P$ is the first integer such that $(2 P-1)(K-1) \geqq t_{i}$.

Note that we may choose $t_{0}=0$ and $t_{1}=1$. In general, if $t_{i} \leqq$ $j_{i}(K-1)$, then the $P$ for $t_{i+1}$ satisfies

$$
2 P-1 \leqq j_{i}+1 \text {. }
$$

Thus, $t_{i+1} \leqq\left(2 j_{i}+1\right)(K-1)$. Thus, if $j_{i} \leqq 2^{i}-1$, we have $j_{i+1} \leqq$ $2^{i+1}-1$.

We now count the number of lines in $A \cup B$. It is less than $2 Q^{2}$. For any given line in $A \cup B$, the number of translates of it through points of $Z^{2}$ which are within distance $M$ of it is at most $2 M / d$, where $d$ is the minimum distance between such translates. If their common slope is $p / q$ in $T$, we have

$$
d \geqq\left(p^{2}+q^{2}\right)^{-1 / 2} \geqq\left(2^{1 / 2} Q\right)^{-1} .
$$

If their common slope with respect to the $y$-axis is in $T$, the mirror image analysis applies. Thus, in all cases, $2 M / d \leqq 2 \cdot 2^{1 / 2} M Q$. Finally, $\left(2 Q^{2}\right)\left(2 \cdot 2^{1 / 2} M Q\right)=4 \cdot 2^{1 / 2} M Q^{3}$ is an upper bound on the number of lines which the subsequence $\left\{z_{t_{i}}\right\}$ can occupy. If the index $i$ on $t_{i}$ is at least $(K-1)\left(4 \cdot 2^{1 / 2} M Q^{3}\right)$, one of these lines will have $K$ points of $\left\{z_{t_{i}}\right\}$. All that is required is that $t_{i} \leqq N$. Since $t_{i} \leqq(K-1)\left(2^{i}-1\right)$, it suffices to have

$$
\log _{2}(K-1)+4 \cdot 2^{1 / 2} M Q^{3}(K-1) \leqq \log _{2} N .
$$

Since $Q=8 \cdot 2^{1 / 2} M(K-1)$, we have $4 \cdot 2^{1 / 2} M Q^{3}(K-1)=2^{13} M^{4}(K-1)^{4}$. By our choice of $N$ this is satisfied. This contradiction establishes the theorem.

REMARK 1. Theorem 1 remains true in $n$-dimensional space 
with the same relations between $N, M$ and $K$ if we use $n-1$ dimensional hyperplanes for $L$ instead of lines. The proof consists of projecting the $S$-walk onto $Z^{2}$, finding a line there and taking its pre-image under the projection.

Remark 2. Professor Carl Pomerance of the University of Georgia [4] has extended this theorem by considering walks whose average step size is bounded. His theorem is stated below. Let $d(V)=\sum_{i=0}^{m-1}\left\|z_{i+1}-z_{i}\right\|$ for a finite sequece $V=\left\{z_{i}\right\}_{i=0}^{m} \subset Z^{2}$.

THEOREM. For every positive integer $K$ and every positive real number $M$, there exists $m_{0}=m_{0}(M, K)$ such that if $m>m_{0}$ and $d(V) / m \leqq M$, then there are $K$ points of $V$ which are collinear.

An effective bound on $m_{0}$ is not known for Pomerance's theorem.

\section{Three dimensional case.}

THEOREM 2. If $S$ is a set of vectors which do not all lie in the same plane, then there exists an infinite S-walk in which no $5^{11}+1$ vectors are collinear.

Notation. If $A=\left(\boldsymbol{a}_{1}, \cdots, \boldsymbol{a}_{n}\right)$ and $B=\left(\boldsymbol{b}_{1}, \cdots, \boldsymbol{b}_{m}\right)$ are ordered sets of vectors, and $\beta$ is a vector operator, we let $R A=\left(\boldsymbol{a}_{n}, \cdots, \boldsymbol{a}_{1}\right)$, $(A, B)=\left(a_{1}, \cdots, a_{n}, b_{1}, \cdots, b_{m}\right)$, and $\beta A=\left(\beta a_{1}, \cdots, \beta a_{n}\right)$. Let $\boldsymbol{i}, \boldsymbol{j}$, and $k$ be the three orthonormal unit vectors. For a vector $z=z_{1} i+$ $z_{2} j+z_{3} k$, let $\|z\|^{\prime \prime}=z_{1}+z_{2}+z_{3}$ and $\|z\|^{\perp}=\left(z_{1}^{2}+z_{2}^{2}+z_{3}^{2}-z_{1} z_{2}-z_{2} z_{3}-\right.$ $\left.z_{3} z_{1}\right)^{1 / 2}$. Note that $\|z\|^{\prime \prime}$ and $\|z\|^{\perp}$ are proportional to the components of $z$ parallel and perpendicular respectively to the vector $i+j+k$. Let $\gamma$ be the length of the component of $i, J$, or $k$ perpendicular to $i+j+k$. Then $\gamma=(2 / 3)^{1 / 2}$ and in general the perpendicular component of $z$ has length $\gamma\|z\|^{\perp}$.

Proof. It suffices to prove Theorem 2 for the case where $S=$ $\{\boldsymbol{i}, \boldsymbol{j}, \boldsymbol{k}\}$. Let $\alpha$ and $\beta$ be vector operators such that $\alpha \boldsymbol{i}=\boldsymbol{j}, \alpha \boldsymbol{j}=\boldsymbol{i}$, $\alpha \boldsymbol{k}=\boldsymbol{k}, \beta \boldsymbol{i}=\boldsymbol{i}, \beta \boldsymbol{j}=\boldsymbol{k}$, and $\beta \boldsymbol{k}=\boldsymbol{j}$. We define inductively ordered sets of vectors $A_{n}$. Let $A_{0}=(i)$, and let $A_{n+1}=\left(A_{n}, \alpha A_{n}, R \beta A_{n}, A_{n}\right.$, $\left.R \beta \alpha A_{n}, R \beta A_{n}, A_{n}\right)$. Note that $A_{n}$ has $7^{n}$ elements and that the sequence $A_{n+1}$ begins with $A_{n}$. It follows that there exists a unique infinite sequence of vectors $\left\{\boldsymbol{v}_{p}\right\}$ such that $\left(\boldsymbol{v}_{1}, \cdots, \boldsymbol{v}_{7^{n}}\right)=A_{n}$ for all $n$. Let $z_{p}=\sum_{q=1}^{p} \boldsymbol{v}_{q}$ for all positive integers $p$. Then $W=\left\{z_{p}\right\}$ is an $S$-walk. We claim that no $5^{11}+1$ elements of $W$ are collinear.

For convenience of notation we let $z_{0}$ be the zero vector. Let $C_{n}^{0}=\left\{z_{0}, z_{1}, \cdots, z_{7^{n}}\right\}$. We prove by induction that the projection of 
$C_{n}^{0}$ onto the plane perpendicular to $i+j+k$ lies within a trapezoid with base $4^{n} \gamma$, base angles $60^{\circ}$, and adjacent sides $4^{n} \gamma / 3$, with $z_{0}$ and $z_{7^{n}}$ lying at extreme ends of the base. We will refer to such a trapezoid as a trapezoid of order $n$. The case $n=0$ is trivial. Assume it is true for $n$. Note that $A_{n}, \alpha A_{n}, R \beta A_{n}$, and $R \dot{\beta} \alpha A_{n}$ are all mirror images of each other, either in space or in time (i.e., one can get from one to the others by permuting the unit vectors, by reversing the order of the sequence, or both). It follows that the set $C_{n}^{\nu}=\left\{z_{7^{n}}, \cdots, z_{7^{n}(\nu+1)}\right\}$ is congruent to $C_{n}^{0}$, or its mirror image, for $0 \leqq \nu \leqq 6$. Therefore the projection of $C_{n}^{\nu}$ lies within a trapezoid of order $n$, with $z_{7^{n} n_{\nu}}$ and $z_{7^{n}(\nu+1)}$ lying at extreme ends of the base. From the definition of $A_{n+1}$, it follows that the seven trapezoids of order $n$ fit together within a trapezoid of order $n+1$, as illustrated in Figure 1.

It is straightforward to prove, by induction on $n$, that for any positive integer $\nu$, the projections of $C_{n+1}^{\nu}$ and $C_{n+1}^{\nu+1}$ can fit together in one of only three possible configurations (ignoring rotations, reflections, and reversals of the sequence), namely those illustrated in Figure 2.

It follows that the distance between two points lying in nonadjacent trapezoids of order $n$ must be at least $3^{-1 / 2} \cdot 4^{n} \gamma$, and that the distance between two points lying in adjacent trapezoids, or

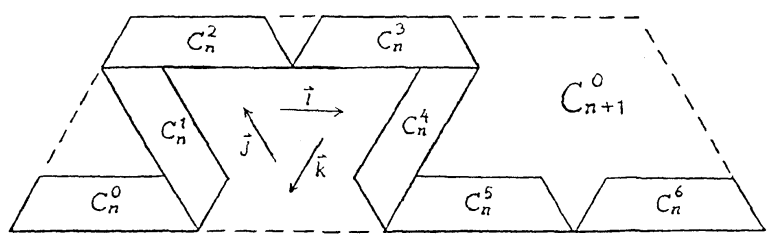

FIGURE 1
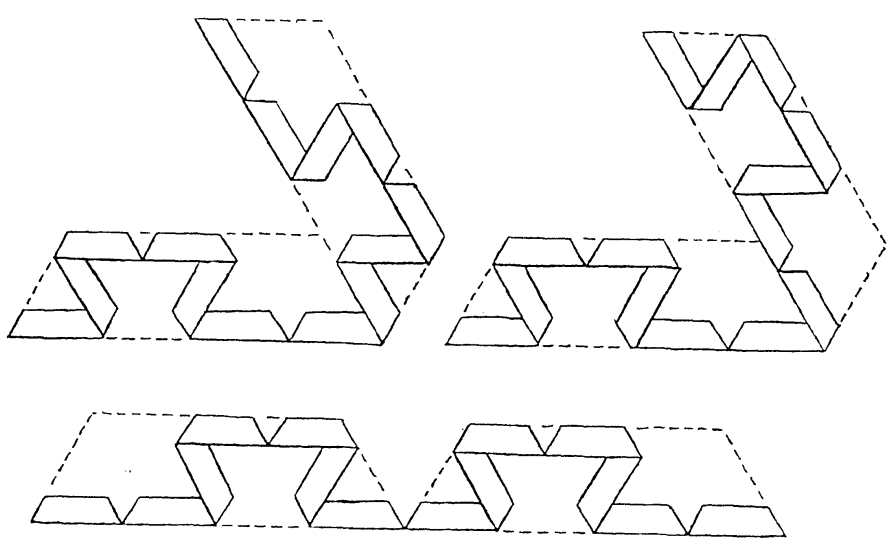

FIGURE 2 
the same trapezoid, of order $n$ can be at most $2 \cdot 4^{n} \gamma$.

Now let $p$ and $q$ be positive integers such that $7^{n} \leqq|p-q|<$ $7^{n+1}$. Then, if $n \geqq 1, z_{p}$ and $z_{q}$ cannot lie in adjacent trapezoids of order $n-1$, so $\left\|z_{p}-z_{q}\right\|^{\perp} \geqq 3^{-1 / 2} \cdot 4^{n-1}$; if $n=0$, this inequality is trivially satisfied. Likewise, $z_{p}$ and $z_{q}$ must lie in adjacent trapezoids, or the same trapezoid, of order $n+1$, so $\left\|z_{p}-z_{q}\right\|^{\perp} \leqq 2 \cdot 4^{n+1}$. Since $\left\|z_{p}-z_{q}\right\|^{\prime \prime}=|p-q|$, we have

$$
3^{-1 / 2} \cdot 4^{n-1} \cdot 7^{-(n+1)}<\left\|z_{p}-z_{q}\right\|^{\perp} /\left\|z_{p}-z_{q}\right\|^{\| \prime} \leqq 2 \cdot 4^{n+1} \cdot 7^{-n} \text {. }
$$

Now let $r$ and $s$ be positive integers such that $7^{m} \leqq|r-s|<7^{m+1}$, with $m \geqq n$, so that

$$
3^{-1 / 2} \cdot 4^{m-1} \cdot 7^{-(m+1)}<\left\|z_{r}-z_{s}\right\|^{\perp} /\left\|z_{r}-z_{s}\right\|^{\prime \prime} \leqq 2 \cdot 4^{m+1} \cdot 7^{-m} .
$$

If $z_{p}, z_{q}, z_{r}$, and $z_{s}$ are collinear, then

$$
\left\|z_{p}-z_{q}\right\|^{\perp} /\left\|z_{p}-z_{q}\right\|^{\| \prime}=\left\|z_{r}-z_{s}\right\|^{\perp} /\left\|z_{r}-z_{s}\right\|^{\prime \prime}
$$

So $3^{-1 / 2} \cdot 4^{n-1} \cdot 7^{-(n+1)}<2 \cdot 4^{m+1} \cdot 7^{-m}$. It follows that $(7 / 4)^{m-n}<224 \sqrt{3}$, and $m-n<(\log 224 \sqrt{3}) /(\log 7 / 4)<11$, i.e., $m-n \leqq 10$. Therefore $|r-s| /|p-q|<7^{11}$, and there are at most $7^{11}$ collinear points in $W$.

Furthermore, if $X$ is a set of collinear points in $W$ which all lie within the same trapezoid of order $n$, but not within the same trapezoid of order $n-1$, then no two points of $X$ can lie within the same trapezoid of order $n-11$. However, no line can intersect more than five trapezoids of order $n-1$ within a trapezoid of order $n$. For suppose a line intersected six of the trapezoids $C_{n}^{0}, C_{n}^{1}, \cdots, C_{n}^{6}$ in Figure 1. If $C_{n}^{0}$ where excluded, then the line would have to intersect $C_{n}^{3}$ and $C_{n}^{5}$, in which case $C_{n}^{1}$ would be missed. If $C_{n}^{2}$ were excluded, then the line would intersect $C_{n}^{0}$ and $C_{n}^{6}$, missing $C_{n}^{3}$. But a line intersecting $C_{n}^{0}$ and $C_{n}^{2}$ would miss $C_{n}^{6}$. Therefore, there are at most $5^{11}$ collinear points in $W$, and the theorem is proved.

It is obvious that this result can be sharpened considerably without changing the method of proof. For example it is not hard to convince oneself, by studying Figure 2, that in fact $4^{n-1} \leqq \| z_{p}$ $z_{q} \|^{\perp} \leqq 4^{n+1}$ if $7^{n} \leqq|p-q|<7^{n+1}$. Also, there is no need to lump together all values of $|p-q|$ between $7^{n}$ and $7^{n+1}$. By using a finer partition it ought to be possible to show that for a given value of $|p-q|$, the possible values of $\left\|z_{p}-z_{q}\right\|^{\perp} /\left\|z_{p}-z_{q}\right\|^{\prime \prime}$ range over a factor no greater than 4 . Since $4<(7 / 4)^{3}$, this would imply that $W$ can have no more than $7^{3}$ collinear points, all lying in the same trapezoid of order $n$, and no two lying in the same trapezoid of order $n-4$. Finally, one could examine the $7^{4}$ trapezoids of order $n-4$ within a trapezoid of order $n$, preferably with the aid of a 
computer, and find an upper bound on the number which can be collinear, not only in the plane, but in 3-space. To clinch the argument, it might be necessary to descend to order $n-5$.

One would hope that by this method a sufficiently clever and persistent mathematician could determine the true maximum number of collinear points in $W$, which undoubtedly is three. However, there is no hope of sharpening Theorem 2 further than this, for we have the following theorem:

THEOREM 3. If $S$ has exactly three elements, then every S-walk of length nine has three collinear vectors; in fact three equally spaced collinear vectors.

Proof. This result follows from the theorem of T. C. Brown [1] that any sequence of length nine on three symbols contains two adjacent segments which are permutations of each other. Brown's theorem can be verified in about one hour by direct computation.

An $S$-walk of length eight with no three collinear points is obtained by summing the sequence $i, j, i, k, i, j, i$.

REMARK 3. Theorem 2 also holds in the case where $S \subset \boldsymbol{R}^{2}$, provided that there are three elements $e_{1}, e_{2}$, and $e_{3}$ of $S$, such that $\boldsymbol{e}_{1} \times \boldsymbol{e}_{2}, \boldsymbol{e}_{2} \times \boldsymbol{e}_{3}$, and $\boldsymbol{e}_{3} \times \boldsymbol{e}_{1}$ are linearly independent over the rationals. In other words, the condition that the elements of $S$ be lattice points is necessary for Theorem 1.

The above theorems leave unanswered the question of whether it is possible to have an infinite $S$-walk with no three collinear points for some $S \subset Z^{n}$ (in particular, can $n=3$ ?).

\section{REFERENCES}

1. T. C. Brown, Is there a sequence on four symbols in which no two adjacent segments are permutations of one another, Amer. Math. Monthly, 78 (1971), 886-888.

2. J. W. S. Cassels, An Introduction to Diophantine Approximations, Cambridge University Press, Cambridge, 1957.

3. P. L. Montgomery, Solution of a problem of T. C. Brown, Amer. Math. Monthly, 79 (1972), 1143-1144.

4. Carl Pomerance, Collinear subsets of lattice point sequences - an analogue of Szemeredi's theorem, to appear in J. Combinatorial Theory.

5. L. Thomas Ramsey, Fourier-Stieljes transforms of measures with a certain continuity property, J. Functional Analysis, 25 (1977), 306-313.

Received February 28, 1978.

UNIVERSITY OF HAWAII

HONOLULU, HI 96822 



\title{
PACIFIC JOURNAL OF MATHEMATICS
}

\section{EDITORS}

DONALD BABBITT (Managing Editor)

University of California

Los Angeles, CA 90024

\section{HUGO RossI}

University of Utah

Salt Lake City, UT 84112

C. C. MOORE and ANDREW OGG

University of California

Berkeley, CA 94720

\section{J. DUGUNDJI}

Department of Mathematics University of Southern California Los Angeles, CA 90007

R. FINN and J. Milgram

Stanford University Stanford, CA 94305

\section{ASSOCIATE EDITORS}
E. F. BECKENBACH
B. H. NeUmanN
F. WOLF
K. YosHIDA

\section{SUPPORTING INSTITUTIONS}

\author{
UNIVERSITY OF BRITISH COLUMBIA \\ CALIFORNIA INSTITUTE OF TECHNOLOGY \\ UNIVERSITY OF CALIFORNIA \\ MONTANA STATE UNIVERSITY \\ UNIVERSITY OF NEVADA, RENO \\ NEW MEXICO STATE UNIVERSITY \\ OREGON STATE UNIVERSITY \\ UNIVERSITY OF OREGON
}

\author{
UNIVERSITY OF SOUTHERN CALIFORNIA \\ STANFORD UNIVERSITY \\ UNIVERSITY OF HAWAII \\ UNIVERSITY OF TOKYO \\ UNIVERSITY OF UTAH \\ WASHINGTON STATE UNIVERSITY \\ UNIVERSITY OF WASHINGTON
}

The Supporting Institutions listed above contribute to the cost of publication of this Journal, but they are not owners or publishers and have no responsibility for its content or policies.

Mathematical papers intended for publication in the Pacific Journal of Mathematics should be in typed form or offset-reproduced, (not dittoed), double spaced with large margins. Please do not use built up fractions in the text of the manuscript. However, you may use them in the displayed equations. Underline Greek letters in red, German in green, and script in blue. The first paragraph or two must be capable of being used separately as a synopsis of the entire paper. Please propose a heading for the odd numbered pages of less than 35 characters. Manuscripts, in triplicate, may be sent to any one of the editors. Please classify according to the scheme of Math. Reviews, Index to Vol. 39. Supply name and address of author to whom proofs should be sent. All other communications should be addressed to the managing editor, or Elaine Barth, University of California, Los Angeles, California, 90024.

50 reprints to each author are provided free for each article, only if page charges have been substantially paid. Additional copies may be obtained at cost in multiples of 50 .

The Pacific Journal of Mathematics is issued monthly as of January 1966. Regular subscription rate: $\$ 84.00$ a year (6 Vols., 12 issues). Special rate: $\$ 42.00$ a year to individual members of supporting institutions.

Subscriptions, orders for numbers issued in the last three calendar years, and changes of address should be sent to Pacific Journal of Mathematics, P.O. Box 969, Carmel Valley, CA 93924, U.S.A. Older back numbers obtainable from Kraus Periodicals Co., Route 100, Millwood, NY 10546.

PUBLISHED BY PACIFIC JOURNAL OF MATHEMATICS, A NON-PROFIT CORPORATION

Printed at Kokusai Bunken Insatsusha (International Academic Printing Co., Ltd.). 8-8, 3-chome, Takadanobaba, Shinjuku-ku, Tokyo 160, Japan.

Copyright (C) 1979 by Pacific Journal of Mathematics Manufactured and first issued in Japan 


\section{Pacific Journal of Mathematics}

\section{Vol. 83, No. $2 \quad$ April, 1979}

Patrick Robert Ahern, On a theorem of Hayman concerning the derivative of a

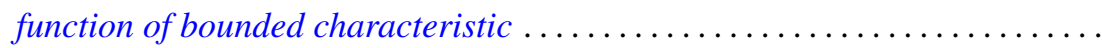

Walter Allegretto, Finiteness of lower spectra of a class of higher order elliptic

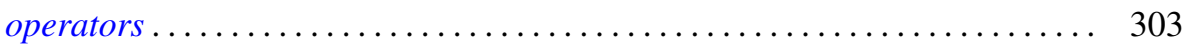

Leonard Asimow, Superharmonic interpolation in subspaces of $C_{c}(X) \ldots \ldots 11$

Steven F. Bellenot, An anti-open mapping theorem for Fréchet spaces . . . . . . . 325

B. J. Day, Locale geometry. . . . . . . . . . . . . . . . . . . . . . . . . . 333

John Erik Fornaess and Steven Krantz, Continuously varying peaking

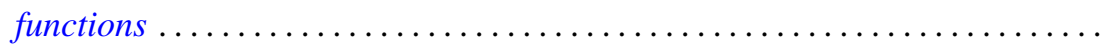

Joseph Leonide Gerver, Long walks in the plane with few collinear points ......

Joseph Leonide Gerver and Lawrence Thom Ramsey, On certain sequences of

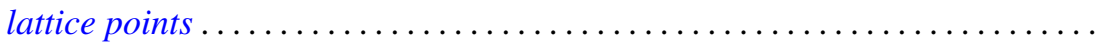

John R. Graef, Yuichi Kitamura, Takaŝi Kusano, Hiroshi Onose and Paul Winton

Spikes, On the nonoscillation of perturbed functional-differential

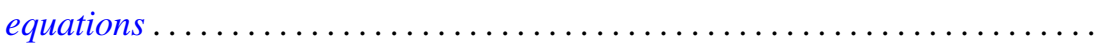

James A. Huckaba and James M. Keller, Annihilation of ideals in commutative

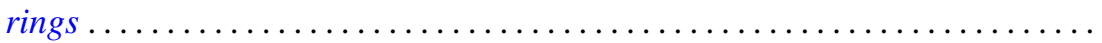

Anzelm Iwanik, Norm attaining operators on Lebesgue spaces . . . . . . . . . . .

Surjit Singh Khurana, Pointwise compactness and measurability . . . .......... 387

Charles Philip Lanski, Commutation with skew elements in rings with

involution.

Hugh Bardeen Maynard, A Radon-Nikodým theorem for finitely additive bounded

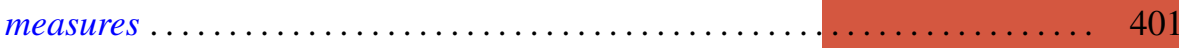

Kevin Mor McCrimmon, Peirce ideals in Jordan triple systems ..

Sam Bernard Nadler, Jr., Joseph E. Quinn and N. Stavrakas, Hyperspaces of compact convex sets.

Ken Nakamula, An explicit formula for the fundamental units of a real pure

sextic number field and its Galois closure ............

Vassili Nestoridis, Inner functions invariant connected components . .

Vladimir I. Oliker, On compact submanifolds with nondegenerate parallel

normal vector fields.

Lex Gerard Oversteegen, Fans and embeddings in the plane.

Shlomo Reisner, On Banach spaces having the property G.L

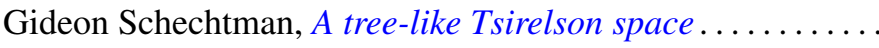

Helga Schirmer, Fix-finite homotopies . . . . . . . . . . . .

Jeffrey D. Vaaler, A geometric inequality with applications to linear forms . .

William Jennings Wickless, $T$ as an $\mathscr{G}$ submodule of $G$.....

Kenneth S. Williams, The class number of $Q(\sqrt{-p})$ modulo 4 , for $p \equiv 3$ (

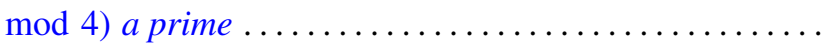

